\title{
Using Reference Point as Feature for Fingerprint Indexing
}

\author{
Alfredo Muñoz-Briseño, Andrés Gago-Alonso, and José Hernández-Palancar \\ Advanced Technologies Application Center (CENATAV), Cuba \\ $\{$ amunoz, agago, jpalancar\}@cenatav.co.cu
}

\begin{abstract}
This work introduces a new feature based on relative minutia position regarding a reference point. The introduction of this feature, allows the elimination of false matches generated by minutiae. Moreover, a novel algorithm for detecting the reference point in fingerprints is introduced. This approach was tested in a manually edited dataset and it proved to be highly tolerant to distorted impressions. Moreover, the new feature was integrated to a recent fingerprint indexing algorithm in an efficient way. Well known fingerprint datasets were employed to show the improvement in accuracy and the superiority of the presented method regarding other proposals.
\end{abstract}

\section{Introduction}

The most commonly used technique in biometric systems is the comparison of fingerprints. The ridge patterns found in fingers and other parts of the human body provide enough information to distinguish a specific person from the rest. In this sense, the purpose of identification is to establish the identity of a specific person, given a query impression and a dataset of fingerprints from different individuals. Modern fingerprint collections usually have millions of entries. Therefore, a reduction of the search space is very convenient in identification algorithms. There are some existing approaches in the literature which try to reduce the search space by using fingerprint indexing [1 5]. This technique offers great advantages because it allows the reduction of the search space with minimal lost of accuracy. The general scheme for describing almost every indexing algorithm is made up of an indexing stage and a retrieving stage. The first one is executed while the fingerprint collection is preprocessed and enrolled. The queries are dealt in a retrieving stage, detecting the query occurrence in the fingerprint collection.

Diverse approaches of indexing algorithms are found in the state of the art. One of the most commonly used feature selection strategies is focused on choosing triplets of minutiae. In some of these approaches, sets of triangles are calculated including, in each one of them, geometric and fingerprint features [4, 2]. Other approaches use information of the minutiae vicinities [6] or the orientation image 7] in order to obtain the features.

In this work, a new minutia feature focused on improving the accuracy of the indexing stage is proposed. Also, a method for fingerprint reference point

E. Bayro-Corrochano and E. Hancock (Eds.): CIARP 2014, LNCS 8827, pp. 367-374 2014.

(C) Springer International Publishing Switzerland 2014 
localization required to obtain such feature is presented. This method uses a combination of some very effective heuristics with the well known Poincare index algorithm. These heuristics are based on the reconstructed orientation image and the maximum curvature points extracted from the fingerprints.

The rest of this paper is organized as follows. In section 2, the new reference point localization algorithm is introduced. In section [3, the minutiae feature is defined. Next, section 4 describes how the new feature is introduced in an indexing algorithm. Finally, experimental results are presented in section 5, and conclusions are given in section 6 .

\section{Reference Point Localization}

In the state of the art, there are many methods of singular points localization based on Poincare index [8]. However, the performance of these approaches report some drawbacks in noisy or bad quality images, since false singularities can be detected. Another handicap for using this method is the lack of singular points in fingerprint classified as archs. The present proposal combines the Poincare index method with some strategies to locate specific patterns in the orientation image. Also, a heuristic to locate the unique reference point is developed.

In many fingerprint verification and identification methods, the blocked orientation image is used with diverse goals. This image is commonly represented as a matrix $O I$ whose elements encode the local orientation of the ridges found in each block of the original impression [8]. There are many situations that may produce a degradation in the values of the $O I$ matrix. The excessive inking of the fingers or overpressure exerted on the surface in which the fingerprint is collected are examples of this fact. These situations produce bad quality zones in which the values of the orientation image are extremely inaccurate. For this reason, it is very important to have a preprocessing strategy dealing with the possible noise in $O I$, since our proposal uses the $O I$ matrix extensively.

As first step of the method, the blocks of $O I$ contained in bad quality zones are located. To perform this task, a method already described in the literature based on the coherence map [8] is used. In these zones, a reconstruction of the orientation map is performed, using a variation of an algorithm proposed by

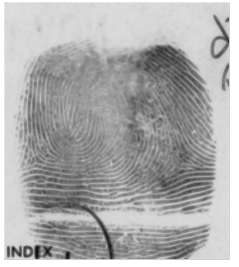

(a) Fingerprint

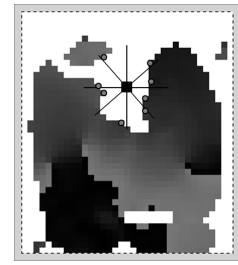

(b) $O I$ with bad quality areas

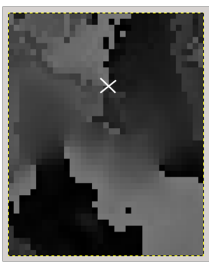

(c) Reconstructed $O I$

Fig. 1. Reconstruction of $O I$ 
Feng and Jain [9]. In this approach, the plane around each bad quality block $b_{i, j}$ is split into $K$ regions of the same area. The value of $b_{i, j}$ is predicted using the orientations $\alpha_{k}$ of the nearest minutia in each region, using the following expression:

$$
b_{i, j}=\frac{1}{2} \arctan \frac{\sum_{k=1}^{K} \cos \left(2 \alpha_{k}\right) w_{k}}{\sum_{k=1}^{K} \sin \left(2 \alpha_{k}\right) w_{k}} .
$$

where $w_{k}$ is a weighting function. As novelty, a variation is introduced by taking as $\alpha_{k}$ the value of the nearest good quality block, instead of the nearest minutia, in region $k$ around $b_{i, j}$ in the orientation image.

In Figure 1(a), a noisy image of a fingerprint is illustrated while, in Figure 1(b) the corresponding orientation image is shown, without the values of the bad quality blocks. In Figure 1(c), the final reconstructed orientation image with the process previously described, is illustrated. For instance, in Figure 1(b), the values used to interpolate the orientation value in the block marked with a square are indicated with a circle. It is important to mention that the method used to compute $O I$ in good quality zones is the implemented by VeriFinger 4.2 [10].

In this approach, the core in convex ridges is used as reference point $p(x, y)$. Due to the structure of the ridges in fingerprints, there are sudden changes in the orientation values of the vicinities of $p(x, y)$. Using this property, we define as points of upper orientation change (UOC) the ones having these changes in specific directions.

In Algorithm 1, a recursive subroutine for computing the amount of blocks with difference of orientation higher than $\phi$, over some coordinates in $O I$ is shown. If OrientDiff $(x, y, O I)>\delta$, then $p(x, y)$ is considered a point of UOC, where $\phi$ and $\delta$ are predefined thresholds.

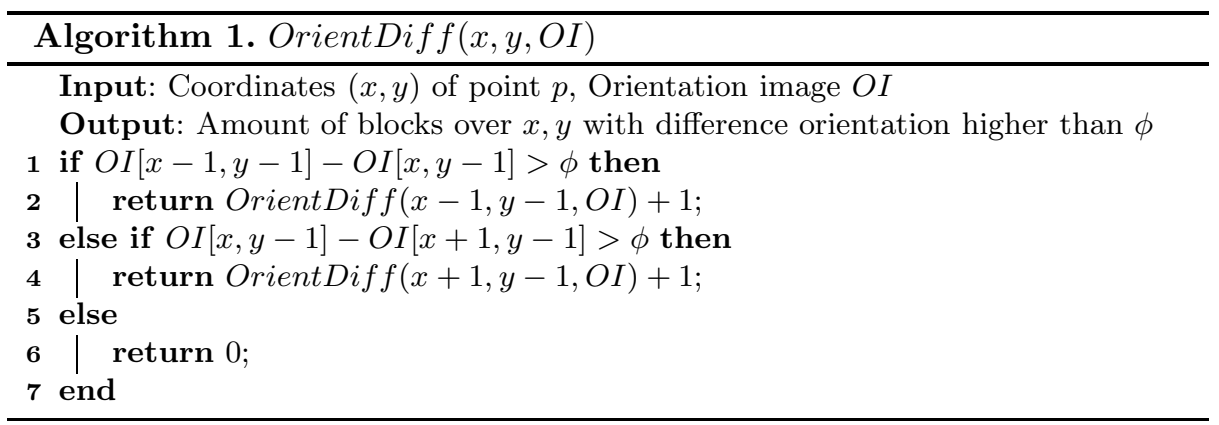

The present proposal uses a variant of the Poincare index method, to build a candidates set $P_{c}=\left\{p_{0}, \ldots, p_{n}\right\}$ to be reference points. This method takes into account the difference of orientations around a block in order to locate cores and deltas. Candidates are only the cores that are also points of UOC. The centroid or barycenter $C_{f}$ of the minutiae extracted from the fingerprint is 


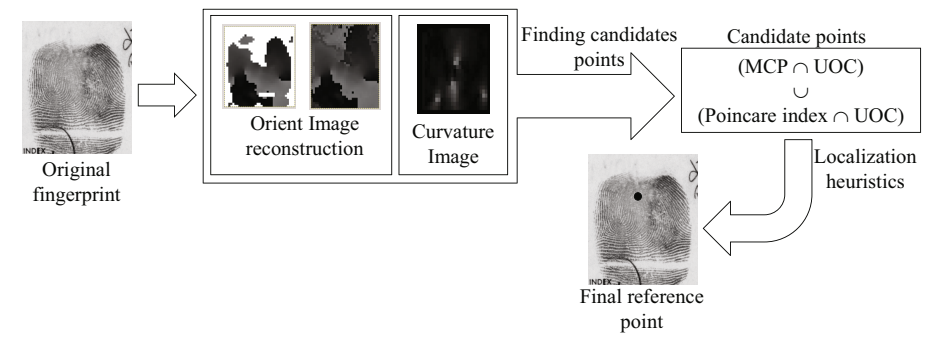

Fig. 2. Flow for the fingerprint reference point localization

also computed. The final reference point is the $p_{i} \in P_{c} \operatorname{such}$ that $\operatorname{dist}\left(p_{i}, C_{f}\right) \leq$ $\operatorname{dist}\left(p_{j}, C_{f}\right), \forall j, 0 \leq j \leq n$, where $\operatorname{dist}()$ is the euclidean distance between two points. This heuristic guarantees that the reference point found will be located as close as possible to the center of the impression.

The Poincare index method is highly affected by noise in the orientation image. Even in some cases, the reconstruction of $O I$ is not enough in order to build the candidate set $P_{c}$. Also, the fingerprints classified as archs do not have singular points. For this reason, the previously introduced procedure may fail in some impressions. In these cases, the set $P_{c}$ is also made by the maximum curvature points (MCP) that are also of upper orientation changes in the orientation image. The MCP are obtained from the curvature image extracted from the fingerprint. A method proposed in the literature is used in order to compute the curvature image [11]. In the same way, the final reference point will be the closest element of $P_{c}$ to $C_{f}$.

The new exposed method is very robust to distortions in the orientation image as is evidenced in Figure 1. Even when some areas in the shown fingerprint are seriously distorted, this algorithm is able to find accurately the reference point. In Figure 2 the complete flow of the proposal for fingerprint reference point localization is illustrated.

\section{Reference Point Feature}

In some fingerprint indexing algorithms [2], it is common to find erroneous matches between features located in different places of the impressions. In Figure $3(\mathrm{a})$, we can see two impressions of a same finger with similar indices found in different areas of the fingerprints. A feature with the capability of providing enough information about the relative position of every minutia would avoid this false match.

With this motivation, a new feature of minutiae named reference point feature, is introduced in the present work. For calculating this feature, the reference point of the fingerprint is computed using the previously defined method (See section 2 ). Then, the area around a minutia $m_{i}$ is split into two concentric disks similar to the method presented by Tico and Kuosmanen [12]. Each one of these disks is also partitioned into regions of the same area, with a label assigned. A label 
is assigned to the area out of the proposed subdivision (label $L_{8}$ in Figure 3(b) . Using the minutia direction as reference, the regions on each disk can be placed and ordered in a counterclock manner. Finally, the new feature $\rho\left(m_{i}\right)$ is given by the label of the region containing the reference point of the fingerprint.

In their work, Tiko and Kuosmanen [12] use concentric disk to select sampling orientation points that conform a descriptor. In our case, a similar strategy of partition is used with a different goal. A position relation between each minutia and a reference point found in the fingerprint is established.

The number of regions in which each disk is split, is inversely proportional to its distance of the minutia. The feature is defined in this way since the distortions between a minutia and the reference point is higher when they are farther away. This situation occurs because the human skin is elastic; thus two impressions of the same finger can have a displacement of some minutiae. That is why the width of the disks, denoted as $w_{1}$ and $w_{2}$ plays an important roll in the impact of the feature. In Figure 3(b), it is shown the labeled subdivision on the vicinity of a minutia. In this case, $\rho\left(m_{i}\right)=L_{7}$.

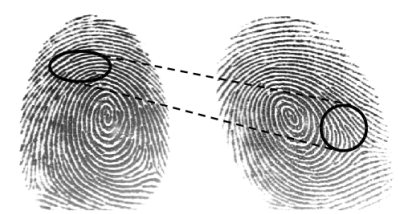

(a)

Erroneous correspondences.

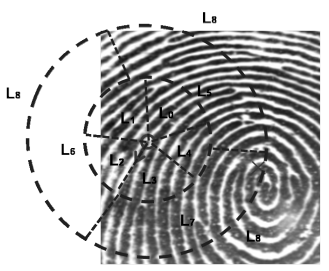

(b) New feature.

Fig. 3. Erroneous correspondences found and feature proposed

\section{Indexing and Retrieving}

In indexing and retrieving steps a recently published approach [2] was improved, by incorporating the minutia feature presented. This proposal uses Delaunay triangles with higher order than 1 in order to build a set of triangles that will represent the fingerprints. This triangle set is defined as:

$$
E T_{k}(P)=T D_{0}(P) \bigcup T D_{k}(P)
$$

where $P$ is a set of points that represents the minutiae extracted from the fingerprints. $T D_{0}(P)$ and $T D_{k}(P)$ are the Delaunay triangulation of order 0 and $k$, respectively. Additionally, the triangles of $E T_{k}(P)$, whose edges cross more than $r$ pixels of bad quality zones, are eliminated. In this way, the number of triangles of $E T_{k}(P)$ remains linear with respect to the amount of minutiae.

In order to perform the indexing stage a feature vector $f\left(\triangle p_{i} p_{j} p_{k}\right)=$ $\left(s_{t}, a_{1}, a_{2}, a_{3}, c_{i j}, c_{j k}, c_{k i}, \rho_{i}, \rho_{j}, \rho_{k}\right)$ is extracted from each triangle $\triangle p_{i} p_{j} p_{k}$ of $E T_{k}(P)$ conformed by the following features: 
- $s_{t}$ : triangle sign. $s_{t}=0$ if the expression $x_{i}\left(y_{j}-y_{k}\right)+x_{j}\left(y_{k}-y_{j}\right)+x_{k}\left(y_{i}-y_{j}\right)$ is less than 0 , and $s_{t}=1$ otherwise.

$-a_{1}, a_{2}$ and $a_{3}$ : relative directions of the minutiae represented by the points $p_{i}, p_{j}$ y $p_{k}$, with respect to their opposite side in $\triangle p_{i} p_{j} p_{k}$.

$-c_{i j}, c_{j k}$ and $c_{k i}$ : ridge counters between the segments $\left(\overline{p_{i} p_{j}}\right),\left(\overline{p_{i} p_{j}}\right)$ and $\left(\overline{p_{i} p_{j}}\right)$.

$-\rho_{i}, \rho_{j}$ and $\rho_{k}$ : reference point features of the minutiae represented by $p_{i}, p_{j}$ and $p_{k}$.

where $\left(x_{i}, y_{i}\right),\left(x_{j}, y_{j}\right)$ and $\left(x_{k}, y_{k}\right)$ represent the coordinates of the points $p_{i}, p_{j}$ and $p_{k}$, respectively. The ridge counter of a specific segment $\overline{p_{i} p_{j}}$ is defined as the number of ridges of the fingerprint crossed by $\left(\overline{p_{i} p_{j}}\right)$. The representation of these features was done in a very compact way. The triangle sign was represented with only one bit while for $a_{i}, c$ and $\rho$ were employed 3,4 and 4 bits respectively. Finally, a feature vector can be stored in 34 bits by concatenating the binary representation of each one of the components. Each vector obtained from the fingerprints is stored in an index table, denoted as $I$.

In the retrieving stage, all the vectors of the query are generated with the same procedure explained, and correspondences are found between them and those stored in $I$. Every correspondence represents a possible match between a subset of minutiae of the query and a subset from some fingerprint previously stored in the system. The final candidate list is obtained from the amount of these matches found for each fingerprint stored, and their geometric coherence. This method is introduced and explained in detail in a recent proposal [2]. In these stages, the novel aspect with respect to the previous mentioned work [2] is the introduction of a new minutia feature. This difference leads to more descriptive indices, with only a slight increment in the size of their representations.

\section{Experimental Evaluation}

The correct localization of the reference point is vital in the effectiveness of the previously introduced feature. For this reason, an evaluation and validation of the method defined in Section 2 was performed in order to ensure the final accuracy of our proposal. Concerning this, there are no known public fingerprints datasets with the reference points edited. Therefore, a comparison with other state of the art methods is difficult. In our case we made a dataset of 850 degraded fingerprints of different pattern classes to test the accuracy of our reference point localization algorithm. In this dataset, the reference points were manually located by experts.

In table 1, we can see the achieved results with our proposal. The impact of the orientation image reconstruction in the final accuracy is also evidenced in table 1. Also the heuristic method based on the curvature image has a great importance in the effectiveness of the method, since 44 of the 850 fingerprints in the test dataset are archs. In the fingerprints where our method fails, either the quality of the impressions was extremely bad or the reference point was missing in the image. The accuracy reached by the presented method is over $98 \%$; this value is comparable with some of the best reported methods [8]. 
Table 1. Accuracy of the reference point localization algorithm

\begin{tabular}{|c|c|c|}
\hline & Centers found correctly & Accuracy (\%) \\
\hline Proposed approach & 835 & $98,23 \%$ \\
\hline Without curv. heuristic & 791 & $93,05 \%$ \\
\hline Without OI reconst. & 805 & $94,70 \%$ \\
\hline
\end{tabular}

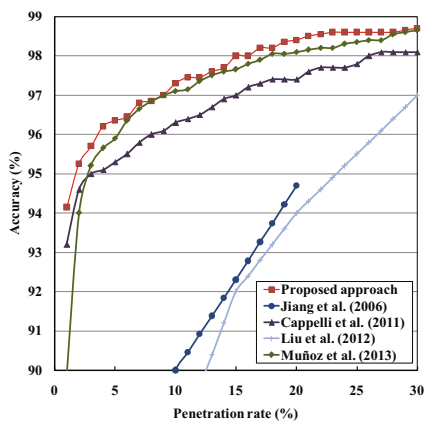

(a) Results in NIST4 dataset.

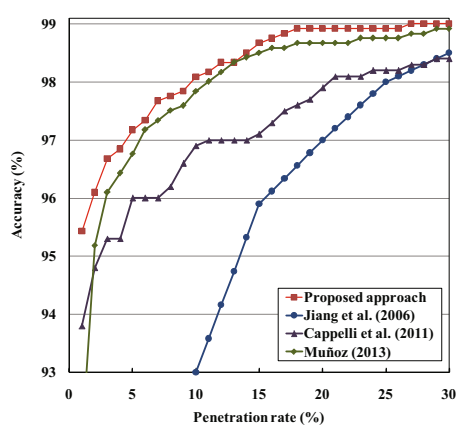

(b) Results in NIST4 natural dataset.

Fig. 4. Resutls in NIST4 dataset

In order to evaluate the impact of the feature introduced in our work, experiments were conducted in the datasets NIST DB4 (4000 rolled impressions from 2000 fingers) and NIST DB4 natural (2408 impressions from 1204 fingers). The selection of these datasets was performed by taking into account that rolled fingerprints are more distorted than plane fingerprints. In this way, we are able to test the new feature under conditions of high distortion and displacement in minutiae. The results reported in the NIST datasets have been obtained by using the first impressions to construct the experimental collection, while the second prints are used as queries to test the indexing performance.

In Figures 4(a) and 4(b) we can see the accuracy of our proposal compared with some of the best reported algorithms, using the trade-off between Penetration Rate and Accuracy. The results reached by Muñoz-Briseño et al. [2] are improved by including the proposed feature, with $w_{1}=30, w_{2}=80, \phi=30$ and $\delta=3$. With these results the positive impact that the new feature has in the elimination of false correspondences, specially for penetration rates lower than $3 \%$, is evidenced. Also, the accuracy reached is superior to the other state-ofthe-art algorithms presented in the experimental evaluation.

\section{Conclusions}

In this paper, a novel minutia feature which allows us to reduce false matches between impressions, was introduced. This feature represents the relative position 
between every minutia and a reference point in the fingerprint. The feature was integrated to an already reported fingerprint indexing algorithm [2] improving its accuracy. The correctness was evaluated in four well known datasets and it was compared with other proposals. Moreover, a new algorithm for locating the reference point was presented and tested in a highly degraded dataset, achieving good results. As future work, the presented reference point localization algorithm will be modified in order to deal with more degraded impressions.

\section{References}

1. Jiang, X., Liu, M., Kot, A.C.: Fingerprint retrieval for identification. IEEE Transactions on Information Forensics and Security 1(4), 532-542 (2006)

2. Muñoz Briseño, A., Gago-Alonso, A., Hernández-Palancar, J.: Fingerprint indexing with bad quality areas. Expert Systems With Applications 40(5), 1839-1846 (2013)

3. Liu, T., Zhu, G., Zhang, C., Hao, P.: Fingerprint indexing based on singular point correlation. In: International Conference on Image Processing, pp. 293-296.

4. Liang, X., Bishnu, A., Asano, T.: A robust fingerprint indexing scheme using minutia neighborhood structure and low-order delaunay triangles. IEEE Transactions on Information Forensics and Security 2(4), 721-733 (2007)

5. Feng, J., Cai, A.: Fingerprint indexing using ridge invariants. In: Proceedings of the 18th International Conference on Pattern Recognition, pp. 433-436 (2006)

6. Cappelli, R., Ferrara, M., Maltoni, D.: Fingerprint indexing based on minutia cylinder-code. IEEE Transactions on Pattern Analysis and Machine Intelligence 33(5), 1051-1057 (2011)

7. Liu, M., Yap, P.T.: Invariant representation of orientation fields for fingerprint indexing. Pattern Recognition 45(7), 2532-2542 (2012)

8. Maltoni, D., Maio, D., Jain, A.K., Prabhakar, S.: Handbook of Fingerprint Recognition, 2nd edn. Springer Publishing Company, Incorporated (2009)

9. Feng, J., Jain, A.K.: Fingerprint reconstruction: From minutiae to phase. IEEE Transactions on Pattern Analysis and Machine Intelligence 33(2), 209-223 (2011)

10. Neurotechnology biometric and artificial intelligence technology (1998-2013), http://www.neurotechnology.com/verifinger.html

11. Qi, J., Xie, M., Wang, W.: A novel fingerprint matching method using a curvaturebased minutia specifier. In: IEEE International Conference on Image Processing, pp. 1488-1491 (2008)

12. Tico, M., Kuosmanen, P.: Fingerprint matching using an orientation-based minutia descriptor. IEEE Transactions on Pattern Analysis and Machine Intelligence 25(8), 1009-1014 (2003) 